\title{
Redshift drift exploration for interacting dark energy
}

\author{
Jia-Jia Geng ${ }^{1}$, Yun-He Li ${ }^{1}$, Jing-Fei Zhang ${ }^{1}$, Xin Zhang ${ }^{1,2, a}$ \\ ${ }^{1}$ Department of Physics, College of Sciences, Northeastern University, Shenyang 110004, China \\ ${ }^{2}$ Center for High Energy Physics, Peking University, Beijing 100080, China
}

Received: 14 February 2015 / Accepted: 25 July 2015 / Published online: 4 August 2015

(C) The Author(s) 2015. This article is published with open access at Springerlink.com

\begin{abstract}
By detecting redshift drift in the spectra of the Lyman- $\alpha$ forest of distant quasars, the Sandage-Loeb (SL) test directly measures the expansion of the universe, covering the "redshift desert" of $2 \lesssim z \lesssim 5$. Thus this method is definitely an important supplement to the other geometric measurements and will play a crucial role in cosmological constraints. In this paper, we quantify the ability of the SL test signal by a CODEX-like spectrograph for constraining interacting dark energy. Four typical interacting dark energy models are considered: (i) $Q=\gamma H \rho_{c}$, (ii) $Q=\gamma H \rho_{d e}$, (iii) $Q=\gamma H_{0} \rho_{c}$, and (iv) $Q=\gamma H_{0} \rho_{d e}$. The results show that for all the considered interacting dark energy models, relative to the current joint $\mathrm{SN}+\mathrm{BAO}+\mathrm{CMB}+H_{0}$ observations, the constraints on $\Omega_{m}$ and $H_{0}$ would be improved by about 60 and 30-40\%, while the constraints on $w$ and $\gamma$ would be slightly improved, with a 30 -year observation of the SL test. We also explore the impact of the SL test on future joint geometric observations. In this analysis, we take the model with $Q=\gamma H \rho_{c}$ as an example, and we simulate future SN and BAO data based on the space-based project WFIRST. We find that with the future geometric constraints, the redshift drift observations would help break the geometric degeneracies in a meaningful way, thus the measurement precisions of $\Omega_{m}, H_{0}, w$, and $\gamma$ could be substantially improved using future probes.
\end{abstract}

\section{Introduction}

Since interactions are ubiquitous in nature, it is rather natural to imagine that dark energy might directly interact with cold dark matter. Actually, that there is no direct interaction at all between dark energy and dark matter is an additional, strong assumption. Currently, one of the most important missions in the field of dark energy research is to provide positive/negative evidence for (i.e., certify/falsify) the scenario

\footnotetext{
a e-mail: zhangxin@mail.neu.edu.cn
}

of interacting dark energy in the light of observational data. Synthetically using the measurements of the expansion history and growth of structure to consistently test the scenario is fairly important. However, for the scenario of interacting dark energy, it is difficult to test models in detail using the measurements of growth of structure due to some complexities, such as the diversity of the construction of covariant 4vector interaction, the large-scale gravity instability, and the lack of abundant, highly accurate data of the growth of structure. Although progress has been made in this aspect since the parametrized post-Friedmann theoretical framework for interacting dark energy was proposed and applied $[1,2]$, an obstacle due to other factors still exists. Under such circumstances, in this work, we only consider to use the geometric measurements to constrain the interacting dark energy models; in particular, we focus on the future redshift drift data.

As a purely geometric measurement, the Sandage-Loeb (SL) test will be crucial to probe the "redshift desert" $(2 \lesssim$ $z \lesssim 5$ ) by directly measuring the expansion of the universe. It was firstly proposed by Sandage [3] to directly measure the variation of redshift of distant sources. Then Loeb [4] found a realistic way of detecting redshift drift in the spectra of Lyman- $\alpha$ forest of distant quasars (QSOs). The 39-m European Extremely Large Telescope (E-ELT) being built was equipped with a high-resolution spectrograph called CODEX (COsmic Dynamics Experiment), which was designed to achieve this goal. Cosmological constraints with the SL test have been studied in numerous works [5-13], some of which simulated 240 or 150 quasars to be observed. However, as pointed out in Ref. [14], only about 30 quasars are bright enough or lying at high enough redshift for actual observation, based on a Monte Carlo simulation using a telescope with a spectrograph like CODEX. Besides, as far as we know, in most existing papers, the best-fit $\Lambda \mathrm{CDM}$ model to the current data is chosen as the fiducial model, based on which SL test data are simulated. Thus, when these SL test data are further combined with other actual data to constrain dark energy models, tension between the simulated SL data and 
other actual data may occur. This is inappropriate and may not give convincing conclusion on the impact of future SL test data on cosmological constraints.

To avoid inconsistency in data, in our previous work [15], we suggested that the best-fit model in the study to current actual data is chosen as the fiducial model in simulating 30 mock SL test data. To give a typical example, we only focused on the dark energy model with constant $w$ (referred to as the $w \mathrm{CDM}$ model). In our recent work [16], we extended the discussion to a time-evolving dark energy model and explored the impact of the SL test data on dark energy constraints in the future geometric measurements.

Though in Ref. [15] a preliminary SL test analysis has been made for two simple interacting dark energy models, a synthetic analysis in depth of quantifying the impact of future redshift drift data on testing different types of interacting dark energy models is still absent. This paper will provide such an analysis. We will quantify the constraining power of future SL test data on different interacting dark energy models, and we will show how the SL test impacts on the parameter estimation.

For interacting dark energy models, the energy balance equations for dark energy and cold dark matter are

$\dot{\rho}_{d e}+3 H \rho_{d e}(1+w)=-Q$,

$\dot{\rho}_{c}+3 H \rho_{c}=Q$,

where $\rho_{d e}$ and $\rho_{c}$ are the background energy densities of dark energy and cold dark matter, respectively. The Hubble parameter $H=\dot{a} / a$ describes the expansion rate of the universe and the interacting term $Q$ describes the energy transfer rate between dark energy and dark matter densities. We consider four typical interacting dark energy models: (i) $Q=\gamma H \rho_{c}$, called the I $w$ CDM1 model, (ii) $Q=\gamma H \rho_{d e}$, called the I $w \mathrm{CDM} 2$ model, (iii) $Q=\gamma H_{0} \rho_{c}$, called the I $w$ CDM3 model, and (iv) $Q=\gamma H_{0} \rho_{d e}$, called the I $w$ CDM4 model. Here $\gamma$ is the dimensionless coupling parameter, and the equation-of-state parameter $w$ is considered to be a constant in this paper.

In fact, when the CODEX experiment is ready to deliver its redshift drift data, other future geometric measurements data will also be available. Therefore, it seems that a more meaningful issue is to ask what role the SL test will play in parameter estimation for interacting dark energy models using the future geometric measurements. To address this issue, we also simulate the future $\mathrm{SN}$ and $\mathrm{BAO}$ data based on the longterm space-based project WFIRST (Wide-Field Infrared Survey Telescope). It is quite common that program in progress is changed or postponed. Besides, because science and technology develop rapidly, it is hard to correctly and truly quantify the percentages of the parameter estimation by SL test data. We only take WFIRST as an example. Owing to the fact that it covers the redshift desert $(2 \lesssim z \lesssim 5)$, the SL test is a valu- able supplementary to other geometric measurements. Moreover, because of the different degeneracy orientations of the SL test and other geometric measurements, it is very credible that the SL test can effectively break the strong degeneracy in other geometric measurements and greatly improve the corresponding cosmological constraint results. It is necessary to point out that this analysis does not entail the necessity of combining the data from WFIRST and CODEX in the future, and that we merely explore the ability of redshift drift to break degeneracy in other geometric measurements and to improve the accuracy of cosmological constraints.

\section{Methodology}

Our procedure is as follows. Interacting dark energy models are first constrained by using the current joint $\mathrm{SN}+\mathrm{BAO}+$ $\mathrm{CMB}+H_{0}$ data, and then for each case the best-fit model is chosen to be the fiducial model in producing the simulated mock SL test data. The obtained SL test data are thus well consistent with the current data. Therefore, it is rather appropriate to combine the mock SL test data with the current data for further constraining the interacting dark energy models. We perform an MCMC likelihood analysis [17] to obtain $\mathcal{O}\left(10^{6}\right)$ samples for each model.

For current data, the most typical geometric measurements are chosen, i.e., the observations of $\mathrm{SN}, \mathrm{BAO}, \mathrm{CMB}$, and $H_{0}$. For the SN data, the SNLS compilation [18] with a sample of $472 \mathrm{SNe}$ is used. For the BAO data, we consider the $r_{s} / D_{V}(z)$ measurements from 6dFGS $(z=0.1)$ [19], SDSS-DR7 $(z=0.35)$ [20], SDSS-DR9 $(z=0.57)$ [21], and WiggleZ ( $z=0.44,0.60$, and 0.73 ) [22] surveys. For the CMB data, we use the Planck distance posterior given by Ref. [23]. As dark energy only affects the CMB through the comoving angular diameter distance to the decoupling epoch (and the late-time ISW effect), the distance information given by the $\mathrm{CMB}$ distance posterior is sufficient for the joint geometric constraint on dark energy. We also use the direct measurement result of the Hubble constant in the light of the cosmic distance ladder from the HST, $H_{0}=73.8 \pm 2.4 \mathrm{~km} \mathrm{~s}^{-1} \mathrm{Mpc}^{-1}$ [24].

Next, we briefly review how to simulate the SL test data. This method is just to directly measure the redshift variation of the quasar Lyman- $\alpha$ absorption lines. The redshift variation is defined as a spectroscopic velocity shift [4],

$\Delta v \equiv \frac{\Delta z}{1+z}=H_{0} \Delta t_{o}\left[1-\frac{E(z)}{1+z}\right]$

where $\Delta t_{o}$ is the time interval of the observation, and $E(z)=$ $H(z) / H_{0}$ is given by specific dark energy models.

According to the Monte Carlo simulations, the uncertainty of $\Delta v$ expected by CODEX can be expressed [14] by 
$\sigma_{\Delta v}=1.35\left(\frac{S / N}{2370}\right)^{-1}\left(\frac{N_{\mathrm{QSO}}}{30}\right)^{-1 / 2}\left(\frac{1+z_{\mathrm{QSO}}}{5}\right)^{x} \mathrm{~cm} \mathrm{~s}^{-1}$

where $S / N$ is the signal-to-noise ratio defined per $0.0125 \AA$ pixel, $N_{\mathrm{QSO}}$ is the number of observed quasars, $z$ QSO represents their redshift, and the last exponent is $x=-1.7$ for $2<z<4$ and $x=-0.9$ for $z>4$.

To simulate the SL test data, we first constrain the interacting dark energy models by using the current data combination. The obtained best-fit parameters are substituted into Eq. (3) to get the central values of the SL test data. We choose $N_{\text {QSO }}=30$ mock SL data uniformly distributed among six redshift bins of $z_{\mathrm{QSO}} \in[2,5]$ and typically take $\Delta t_{o}=30$ $\mathrm{yr}$ in our analysis. The error bars are computed from Eq. (4) with $S / N=3000$.

In order to quantify the power of the SL test in future high-precision joint geometric constraints on dark energy, we simulate future SN and BAO data based on the long-term space-based project WFIRST using the method presented in Ref. [25], and we take the interacting dark energy model with $Q=\gamma H \rho_{c}$ as an example. We simulate 2000 future SNe distributed in 16 bins over the range $z=0.1$ to $z=1.7$. The observables are apparent magnitudes $m_{i}=M+\mu\left(z_{i}\right)$, where $M$ represents the absolute magnitude, and $\mu\left(z_{i}\right)$ is the distance modulus. We also include an additional "near sample" of $500 \mathrm{SNe}$ at $z \approx 0.025$. For future BAO data, we simulate 10000 mock BAO data uniformly distributed over 10 redshift bins of $z \in[0.5,2]$, with each $\Delta z_{i}$ centered on the grid $z_{i}$. The observables are the expansion rate $H(z)$ and the comoving angular diameter distance $d_{A}^{c o}(z)=d_{L}(z) /(1+z)$. For details, we also refer the reader to Ref. [16].

\section{Results and discussion}

First, we constrain the $w \mathrm{CDM}$ model and four typical interacting dark energy models from the current $\mathrm{SN}+\mathrm{BAO}+\mathrm{CMB}$ $+H_{0}$ data combination, and we present the detailed fit results in Table 1. From this table, one can clearly see that for the $w \mathrm{CDM}$ model, $w<-1$ is preferred at about the $1.8 \sigma$ level, while $w<-1$ is preferred at more than $2.2 \sigma$ level for all the four interacting dark energy models. For the $w \mathrm{CDM}$, $\mathrm{I} w \mathrm{CDM} 2, \mathrm{I} w \mathrm{CDM} 3$, and I $w \mathrm{CDM} 4$ models, $\Omega_{c} h^{2}$ can be tightly constrained, and a smaller value is preferred. But for the I $w$ CDM1 model, $\Omega_{c} h^{2}$ cannot be well constrained, and a bigger value is more favored in this case. The coupling $\gamma$ is tightly constrained in the I $w$ CDM 1 model, but its constraint is much weaker in the I $w \mathrm{CDM} 2, \mathrm{I} w \mathrm{CDM} 3$, and $\mathrm{I} w \mathrm{CDM} 4$ models. For the I $w$ CDM 1 model, $\gamma<0$ is preferred at about $2.1 \sigma$ level, while $\gamma<0$ is slightly favored at about $1.4 \sigma$ level for I $w \mathrm{CDM} 2, \mathrm{I} w \mathrm{CDM} 3$, and I $w \mathrm{CDM} 4$ models.

Then we combine the simulated 30-year SL test data with current data and show the constraint results in Figs. 1, 2, and 3. To give an intuitive comparison, the results from current only data are also presented. Figures 1 and 2 show the joint constraints on the $w \mathrm{CDM}, \mathrm{I} w \mathrm{CDM} 1, \mathrm{I} w \mathrm{CDM} 2$, $\mathrm{I} w \mathrm{CDM} 3$, and I $w \mathrm{CDM} 4$ models in the $\Omega_{m}-H_{0}$ and $\Omega_{m}-w$ planes, respectively. Figure 3 shows the joint constraints on the four interacting dark energy models in the $\Omega_{m}-\gamma$ plane. In these three figures, the 68.3 and $95.4 \%$ CL posterior distribution contours are shown. The data combinations used are the current only and the current + SL 30-year combinations, and their constraint results are shown with red and blue contours, respectively. Here we use "current" to denote the current $\mathrm{SN}+\mathrm{BAO}+\mathrm{CMB}+H_{0}$ data combination for convenience. One can clearly see that the degeneracies are well broken with the SL test data for all the models.

The $1 \sigma$ errors of the parameters $w, \gamma, \Omega_{m}$, and $H_{0}$ for the five models with current only and current + SL 30-year data are given in Table 2. With the 30-year SL observation, the constraints on $\Omega_{m}$ and $H_{0}$ will be improved, respectively, by 81.4 and $64.6 \%$ for the $w \mathrm{CDM}$ model, by 68.3 and $27.9 \%$ for the I $w$ CDM1 model, by 58.0 and $44.1 \%$ for the I $w$ CDM2 model, by 58.3 and $44.7 \%$ for the I $w$ CDM3 model, and by 58.3 and $43.3 \%$ for the I $w$ CDM 4 model. Therefore, we can see that with a 30-year observation of the SL test the geometric constraints on dark energy would be improved enormously. For all the considered interacting dark energy models, the constraints on $\Omega_{m}$ and $H_{0}$ would be improved, relative to the current joint observations, by about 60 and $30-40 \%$, with the SL 30-year observation.

Table 1 Fit results for the $w \mathrm{CDM}, \mathrm{I} w \mathrm{CDM} 1, \mathrm{I} w \mathrm{CDM} 2, \mathrm{I} w \mathrm{CDM} 3$, and $\mathrm{I} w \mathrm{CDM} 4$ models using the current $\mathrm{CMB}+\mathrm{BAO}+\mathrm{SN}+H_{0}$ data

\begin{tabular}{|c|c|c|c|c|c|}
\hline Parameter & $w \mathrm{CDM}$ & $\mathrm{I} w \mathrm{CDM} 1$ & $\mathrm{I} w \mathrm{CDM} 2$ & $\mathrm{I} w \mathrm{CDM} 3$ & $\mathrm{I} w \mathrm{CDM} 4$ \\
\hline$\Omega_{b} h^{2}$ & $0.02218_{-0.00029}^{+0.00025}$ & $0.02229_{-0.00024}^{+0.00033}$ & $0.02232 \pm 0.00027$ & $0.02231 \pm 0.00028$ & $0.02230_{-0.00026}^{+0.00030}$ \\
\hline$\Omega_{c} h^{2}$ & $0.1201_{-0.0022}^{+0.0020}$ & $0.1288_{-0.0049}^{+0.0070}$ & $0.1209_{-0.0022}^{+0.0019}$ & $0.1215_{-0.0023}^{+0.0020}$ & $0.1206_{-0.0019}^{+0.0021}$ \\
\hline$w$ & $-1.103 \pm 0.058$ & $-1.136_{-0.055}^{+0.062}$ & $-1.152_{-0.072}^{+0.064}$ & $-1.156_{-0.060}^{+0.070}$ & $-1.149_{-0.077}^{+0.063}$ \\
\hline$\gamma$ & $\cdots$ & $-0.0112_{-0.0078}^{+0.0054}$ & $-0.0284_{-0.0206}^{+0.0218}$ & $-0.0280_{-0.0201}^{+0.0194}$ & $-0.0299_{-0.0264}^{+0.0215}$ \\
\hline$\Omega_{m}$ & $0.2844_{-0.0093}^{+0.0104}$ & $0.2849_{-0.0085}^{+0.0114}$ & $0.2834_{-0.0114}^{+0.0086}$ & $0.2825_{-0.0100}^{+0.0096}$ & $0.2822_{-0.0095}^{+0.0102}$ \\
\hline$H_{0}$ & $70.74_{-1.30}^{+1.26}$ & $72.81_{-1.67}^{+1.87}$ & $71.09_{-1.10}^{+1.39}$ & $71.34 \pm 1.33$ & $71.15_{-1.25}^{+1.27}$ \\
\hline
\end{tabular}



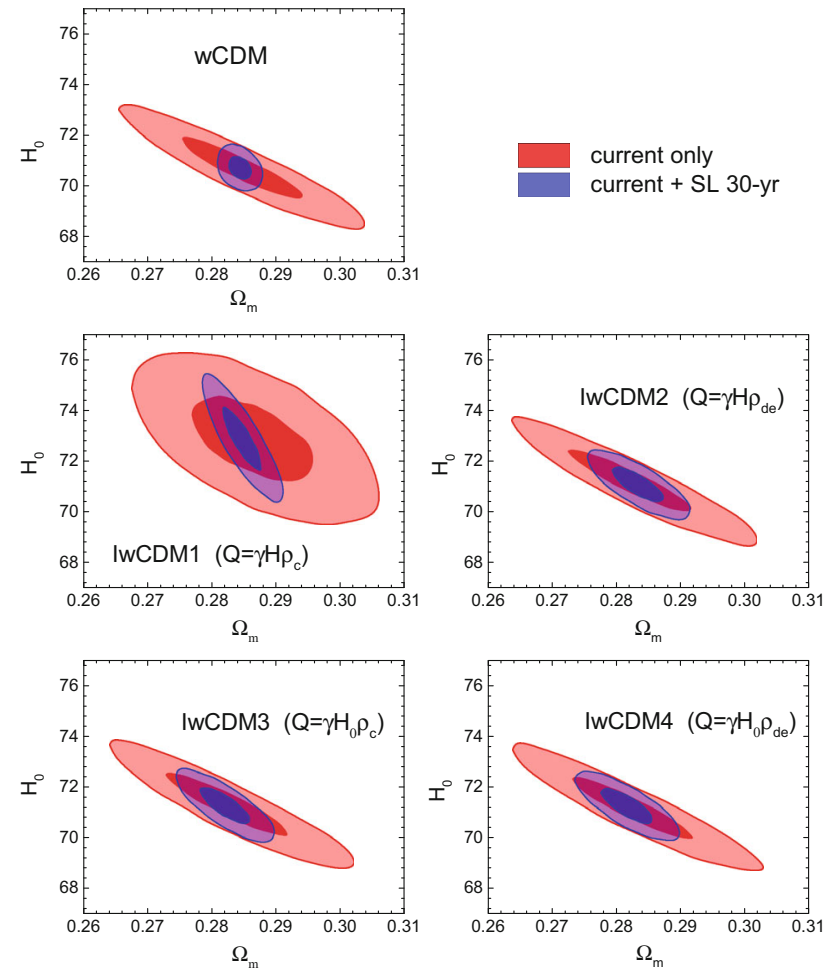

Fig. 1 Constraints ( 68.3 and $95.4 \%$ CL) in the $\Omega_{m}-H_{0}$ plane for the $w \mathrm{CDM}, \mathrm{I} w \mathrm{CDM} 1, \mathrm{I} w \mathrm{CDM} 2, \mathrm{I} w \mathrm{CDM} 3$, and I $w \mathrm{CDM} 4$ models with current only and current + SL 30-year data
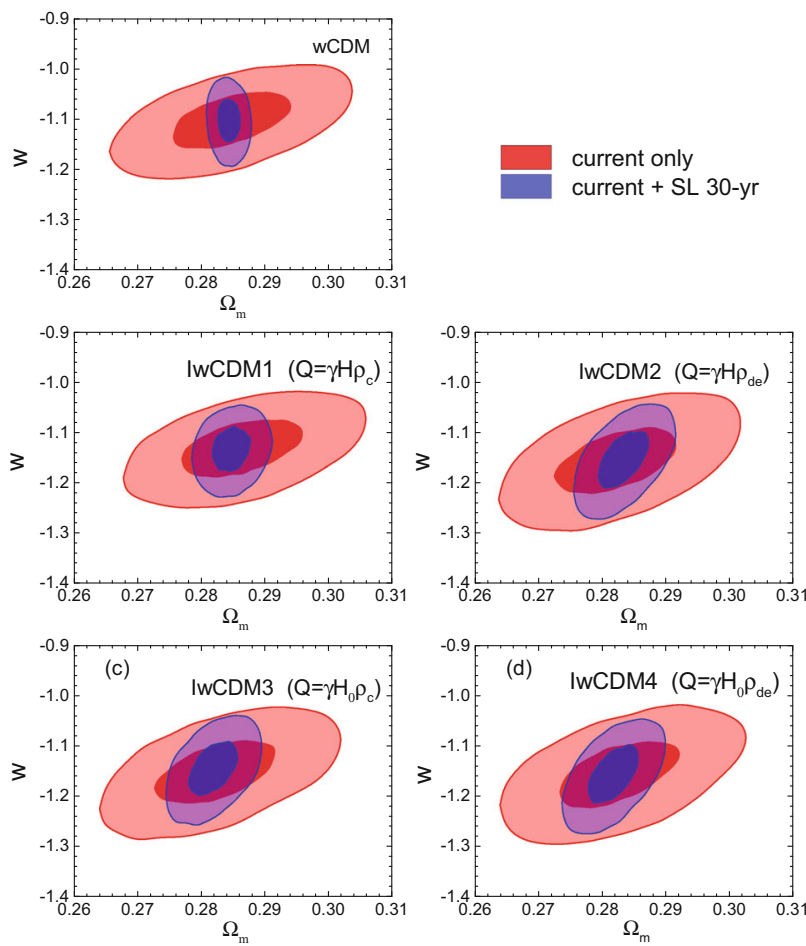

Fig. 2 Constraints ( 68.3 and $95.4 \% \mathrm{CL}$ ) in the $\Omega_{m}-w$ plane for the $w \mathrm{CDM}, \mathrm{I} w \mathrm{CDM} 1, \mathrm{I} w \mathrm{CDM} 2, \mathrm{I} w \mathrm{CDM} 3$, and I $w \mathrm{CDM} 4$ models with current only and current + SL 30-year data

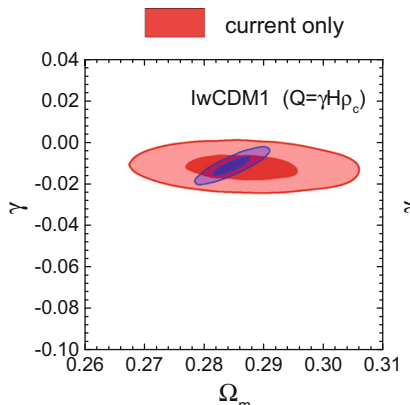

current + SL 30-yr
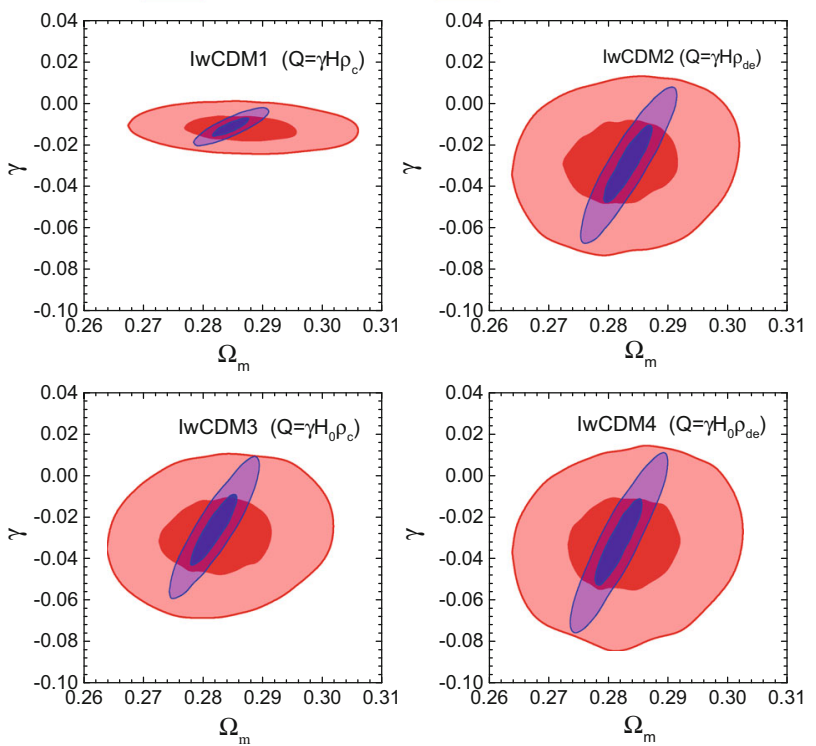

Fig. 3 Constraints $\left(68.3\right.$ and $95.4 \%$ CL) in the $\Omega_{m}-\gamma$ plane for the I $w \mathrm{CDM} 1, \mathrm{I} w \mathrm{CDM} 2, \mathrm{I} w \mathrm{CDM} 3$, and I $w \mathrm{CDM} 4$ models with current only and current + SL 30-year data

We also discuss the impact of the SL test data on constraining the equation of state $w$ and the coupling $\gamma$. The SL 30-year observation helps improve the constraints on $w$ by $24.4,20.5,14.6,10.9$, and $13.1 \%$ for the $w \mathrm{CDM}, \mathrm{I} w \mathrm{CDM} 1$, $\mathrm{I} w \mathrm{CDM} 2, \mathrm{I} w \mathrm{CDM} 3$, and I $w \mathrm{CDM} 4$ models, respectively. The SL 30-year observation helps improve the constraints on $\gamma$ by $30.5,9.0,9.7$ and $8.8 \%$ for the I $w$ CDM1, I $w$ CDM2, $\mathrm{I} w \mathrm{CDM} 3$, and I $w \mathrm{CDM} 4$ models, respectively. Therefore, we find that among the four interacting dark energy models, the I $w$ CDM1 model is the best one in the sense that the constraint results could be improved by the SL test data.

We have discussed the quantification of the impact of future SL test data on constraining interacting dark energy from current $\mathrm{SN}+\mathrm{BAO}+\mathrm{CMB}+H_{0}$ observations. The results show that future SL test data can effectively break the degeneracies in the current data for interacting dark energy models and thus will provide fairly important supplement to the other observations. In the following, we will further explore what role the SL test will play in the future geometric constraints on interacting dark energy. We take the I $w$ CDM1 model (with $Q=\gamma H \rho_{c}$ ) as an example for this analysis and compare the result with that of the $w \mathrm{CDM}$ model. As mentioned above, we simulate future $\mathrm{SN}$ and $\mathrm{BAO}$ data based on the WFIRST mission in this analysis.

Figure 4 shows the joint constraints on the $w \mathrm{CDM}$ model in the $\Omega_{m}-H_{0}$ and $\Omega_{m}-w$ planes. Figure 5 shows the joint constraints on the I $w$ CDM1 model in the $\Omega_{m}-H_{0}, \Omega_{m}-w$, $\Omega_{m}-\gamma$, and $w-\gamma$ planes, respectively. The 68.3 and $95.4 \%$ CL posterior distribution contours are shown. The data combinations used are the future only and the future + SL 30-year 
Table 2 Errors of parameters in the $w \mathrm{CDM}$, I $w \mathrm{CDM} 1$, I $w$ CDM2, I $w \mathrm{CDM} 3$, and I $w \mathrm{CDM} 4$ models for the fits to current only and current $+\mathrm{SL}$ 30-year data

\begin{tabular}{|c|c|c|c|c|c|c|c|c|c|c|}
\hline \multirow[t]{2}{*}{ Error } & \multicolumn{5}{|c|}{ Current only } & \multicolumn{5}{|c|}{ Current + SL 30-year } \\
\hline & $w \mathrm{CDM}$ & $\mathrm{I} w \mathrm{CDM} 1$ & $\mathrm{I} w \mathrm{CDM} 2$ & $\mathrm{I} w \mathrm{CDM} 3$ & $\mathrm{I} w \mathrm{CDM} 4$ & $w \mathrm{CDM}$ & $\mathrm{I} w \mathrm{CDM} 1$ & $\mathrm{I} w \mathrm{CDM} 2$ & $\mathrm{I} w \mathrm{CDM} 3$ & $\mathrm{I} w \mathrm{CDM} 4$ \\
\hline$\sigma(w)$ & 0.082 & 0.083 & 0.096 & 0.092 & 0.099 & 0.062 & 0.066 & 0.082 & 0.082 & 0.086 \\
\hline$\sigma(\gamma)$ & - & 0.0095 & 0.0300 & 0.0279 & 0.0340 & - & 0.0066 & 0.0273 & 0.0252 & 0.0310 \\
\hline$\sigma\left(\Omega_{m}\right)$ & 0.0140 & 0.0142 & 0.0143 & 0.0139 & 0.0139 & 0.0026 & 0.0045 & 0.0060 & 0.0058 & 0.0058 \\
\hline$\sigma\left(H_{0}\right)$ & 1.81 & 2.51 & 1.77 & 1.88 & 1.78 & 0.64 & 1.81 & 0.99 & 1.04 & 1.01 \\
\hline
\end{tabular}
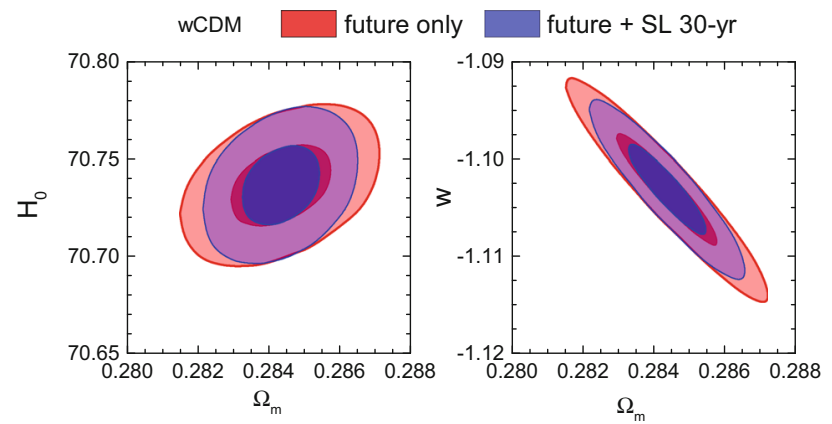

Fig. 4 Constraints $(68.3$ and $95.4 \% \mathrm{CL})$ in the $\Omega_{m}-H_{0}$ plane and in the $\Omega_{m}-w$ plane for $w \mathrm{CDM}$ model with future only and future $+\mathrm{SL}$ 30-year data
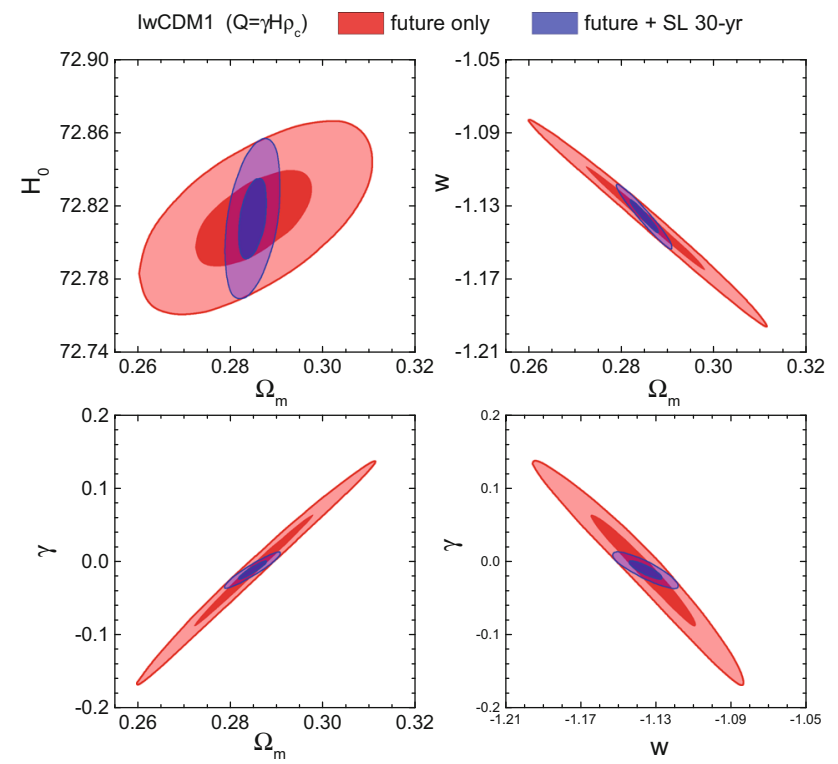

Fig. 5 Constraints $(68.3$ and $95.4 \% \mathrm{CL})$ results for I $w$ CDM1 model with future only and future + SL 30-year data

combinations, and their constraint results are shown with red and blue contours, respectively. The $1 \sigma$ errors of the parameters $w, \gamma, \Omega_{m}$, and $H_{0}$ for the $w \mathrm{CDM}$ model and the I $w \mathrm{CDM} 1$ model for the above data combinations are given in Table 3. Note that here we use "future" to denote the data combination of future $\mathrm{SN}$ and $\mathrm{BAO}$ for convenience. It is shown
Table 3 Errors of parameters in the $w \mathrm{CDM}$ and I $w \mathrm{CDM} 1$ models for the fits to future only and future + SL 30-year data

\begin{tabular}{llllll}
\hline Error & \multicolumn{2}{l}{ Future only } & & \multicolumn{2}{l}{ Future + SL 30 -year } \\
\cline { 2 - 3 } \cline { 6 - 6 } & $w \mathrm{CDM}$ & $\mathrm{I} w \mathrm{CDM} 1$ & & $w \mathrm{CDM}$ & $\mathrm{I} w$ CDM1 \\
\hline$\sigma(w)$ & 0.0083 & 0.0416 & & 0.0067 & 0.0132 \\
$\sigma(\gamma)$ & - & 0.1119 & & 0.0181 \\
$\sigma\left(\Omega_{m}\right)$ & 0.0021 & 0.0190 & & 0.0016 & 0.0045 \\
$\sigma\left(H_{0}\right)$ & 0.0296 & 0.0374 & & 0.0286 & 0.0315 \\
\hline
\end{tabular}

that with the 30-year SL observation, the constraints on $\Omega_{m}$ and $H_{0}$ will be improved by 23.8 and $3.4 \%$ for the $w \mathrm{CDM}$ model, and by 76.3 and $15.8 \%$ for the I $w$ CDM1 model. For the $w \mathrm{CDM}$ model, the constraints on $w$ can be improved by $19.3 \%$, with the SL 30 -year observation, while for the I $w$ CDM1 model, the SL 30-year observation helps improve the constraints on $w$ and $\gamma$ by 68.3 and $83.8 \%$, respectively.

Comparing Figs. 4 and 5, we find that in the future geometric constraints, the redshift drift observation could not break the degeneracies for the $w \mathrm{CDM}$ model, but it could efficiently break the degeneracies for the I $w$ CDM1 model. In the future geometric constraints, for the I $w \mathrm{CDM} 1$ model, the SL 30-year observation would help improve the measurement precisions of $\Omega_{m}, H_{0}, w$, and $\gamma$ by more than 75,15 , 65 , and $80 \%$, respectively.

The main purpose of this work is to investigate the possible impact of future SL test data on existing geometric measurements. In the future, in the case that the $\Lambda \mathrm{CDM}$ model is excluded, one important task is to constrain the coupling parameter $\gamma$, if we wish to find evidence for the existence of interaction between dark sectors. Therefore, it is quite meaningful to investigate the impact of future SL test data on the parameter estimation for interacting dark energy models. For all considered interacting $w \mathrm{CDM}$ models in this work, it is shown that the SL test can effectively break the existing parameter degeneracies and greatly improve the precisions of parameter estimation. The results are consistent with the cases of the $\Lambda \mathrm{CDM}, w \mathrm{CDM}$, and $w_{0} w_{a} \mathrm{CDM}$ models $[15,16]$. By considering more models, we can conclude that the improvement of the parameter estimation by the SL 
test data is independent of the cosmological models in the background. This shows the importance of including SL test data in future cosmological constraints.

\section{Summary}

In this paper, we have analyzed how the redshift drift measurement (i.e. the SL test signal) would have an impact on the parameter estimation for the interacting dark energy models. By detecting the redshift drift in the spectra of the Lyman$\alpha$ forest of distant quasars, the SL test directly measures the expansion rate of the universe in the redshift range of $2 \lesssim z \lesssim 5$, providing an important supplement to other probes in dark energy constraints. We consider four typical interacting dark energy models: (i) $Q=\gamma H \rho_{c}$, (ii) $Q=\gamma H \rho_{d e}$, (iii) $Q=\gamma H_{0} \rho_{c}$, and (iv) $Q=\gamma H_{0} \rho_{d e}$.

Following our previous work $[15,16]$, in order to guarantee that the simulated SL test data are consistent with the other geometric measurement data, we used the best-fitting dark energy models constrained by the current combined geometric measurement data as the fiducial models to produce the mock SL test data.

We showed that the SL test data are extremely helpful in breaking the existing parameter degeneracies. Compared to the current $\mathrm{SN}+\mathrm{BAO}+\mathrm{CMB}+H_{0}$ constraint results, the 30-year observation of SL test could improve the constraints for all the considered interacting dark energy models on $\Omega_{m}$ and $H_{0}$ by about 60 and $30-40 \%$, while the constraints on $w$ and $\gamma$ can be only slightly improved.

We also quantified the impact of the SL test data on the interacting dark energy constraints in the future geometric measurements. To do this analysis, we simulated the future SN and BAO data based on the long-term space-based project WFIRST. We found that the SL test could also play a crucial role in the future joint geometric constraints, especially for the constraints on $w$ and $\gamma$. Taking the interacting dark energy model with $Q=\gamma H \rho_{c}$ as an example, the 30-year observation of the SL test would help improve the measurement precision of $\Omega_{m}, H_{0}, w$, and $\gamma$ by more than $75,15,65$, and $80 \%$, respectively.

Acknowledgments This work was supported by the National Natural Science Foundation of China under Grant No. 11175042, the Provincial Department of Education of Liaoning under Grant No. L2012087, and the Fundamental Research Funds for the Central Universities under Grants No. N140505002, No. N140506002, and No. N140504007.

Open Access This article is distributed under the terms of the Creative Commons Attribution 4.0 International License (http://creativecomm ons.org/licenses/by/4.0/), which permits unrestricted use, distribution, and reproduction in any medium, provided you give appropriate credit to the original author(s) and the source, provide a link to the Creative Commons license, and indicate if changes were made.

Funded by SCOAP ${ }^{3}$.

\section{References}

1. Y.H. Li, J.F. Zhang, X. Zhang, Phys. Rev. D 90, 063005 (2014). arXiv: 1404.5220 [astro-ph.CO]

2. Y.H. Li, J.F. Zhang, X. Zhang, Phys. Rev. D 90, 123007 (2014). arXiv: 1409.7205 [astro-ph.CO]

3. A. Sandage, Astrophys. J. 136, 319 (1962)

4. A. Loeb, Astrophys. J. 499, L111 (1998). arXiv:astro-ph/9802122

5. P.-S. Corasaniti, D. Huterer, A. Melchiorri, Phys. Rev. D 75, 062001 (2007). arXiv:astro-ph/0701433

6. A. Balbi, C. Quercellini, Mon. Not. Roy. Astron. Soc. 382, 1623 (2007). arXiv:0704.2350 [astro-ph]

7. H.-B. Zhang, W. Zhong, Z.H. Zhu, S. He, Phys. Rev. D 76, 123508 (2007). arXiv:0705.4409 [astro-ph]

8. J. Zhang, L. Zhang, X. Zhang, Phys. Lett. B 691, 11 (2010). arXiv: 1006.1738 [astro-ph.CO]

9. Z. Li, K. Liao, P. Wu, H. Yu, Z.-H. Zhu, Phys. Rev. D 88(2), 023003 (2013). arXiv:1306.5932 [gr-qc]

10. S. Yuan, S. Liu and T.-J. Zhang. arXiv:1311.1583 [astro-ph.CO]

11. M. Martinelli, S. Pandolfi, C.J.A.P. Martins, P.E. Vielzeuf, Phys. Rev. D 86, 123001 (2012). arXiv:1210.7166 [astro-ph.CO]

12. J. Darling, Astrophys. J. Lett. 761, L26 (2012). arXiv:1211.4585 [astro-ph.CO]

13. H.-R. Yu, T.-J. Zhang, U.-L. Pen, Phys. Rev. Lett. 113, 041303 (2014). arXiv:1311.2363 [astro-ph.CO]

14. J. Liske, A. Grazian, E. Vanzella, M. Dessauges, M. Viel, L. Pasquini, M. Haehnelt, S. Cristiani et al., Mon. Not. Roy. Astron. Soc. 386, 1192 (2008). arXiv:0802.1532 [astro-ph]

15. J.J. Geng, J.F. Zhang, X. Zhang, JCAP 07, 006 (2014). arXiv: 1404.5407 [astro-ph.CO]

16. J.J. Geng, J.F. Zhang, X. Zhang, JCAP 12, 018 (2014). arXiv:1407.7123 [astro-ph.CO]

17. A. Lewis, S. Bridle, Phys. Rev. D 66, 103511 (2002). arXiv:astro-ph/0205436

18. A. Conley et al. [SNLS Collaboration], Astrophys. J. Suppl. 192, 1 (2011). arXiv:1104.1443 [astro-ph.CO]

19. F. Beutler, C. Blake, M. Colless, D.H. Jones, L. Staveley-Smith, L. Campbell, Q. Parker, W. Saunders et al., Mon. Not. Roy. Astron. Soc. 416, 3017 (2011). arXiv:1106.3366 [astro-ph.CO]

20. N. Padmanabhan, X. Xu, D.J. Eisenstein, R. Scalzo, A.J. Cuesta, K.T. Mehta, E. Kazin, Mon. Not. Roy. Astron. Soc. 427(3), 2132 (2012). arXiv:1202.0090 [astro-ph.CO]

21. L. Anderson, E. Aubourg, S. Bailey, D. Bizyaev, M. Blanton, A.S. Bolton, J. Brinkmann, J.R. Brownstein et al., Mon. Not. Roy. Astron. Soc. 427(4), 3435 (2013). arXiv:1203.6594 [astro-ph.CO]

22. C. Blake, S. Brough, M. Colless, C. Contreras, W. Couch, S. Croom, D. Croton, T. Davis et al., Mon. Not. Roy. Astron. Soc. 425, 405 (2012). arXiv:1204.3674 [astro-ph.CO]

23. Y. Wang, S. Wang, Phys. Rev. D 88, 043522 (2013). arXiv:1304.4514 [astro-ph.CO]

24. A.G. Riess et al., Astrophys. J. 730, 119 (2011). arXiv:1103.2976 [astro-ph.CO]

25. A. Albrecht, G. Bernstein, R. Cahn, W.L. Freedman, J. Hewitt, W. Hu, J. Huth and M. Kamionkowski et al. arXiv:astro-ph/0609591 